\title{
Ethnologies
}

\section{Regard sur... les jeunes en Europe centrale et orientale. Par Mircea Vultur, dir. (Québec : Presses de l'Université Laval et Éditions de l'IQRC, 2004. Pp. 142, ISBN 2-89224-347-5)}

\section{Cezar Aurel Banu}

Volume 28, numéro 2, 2006

Les noces en vrai

Wedding Realities

URI : https://id.erudit.org/iderudit/014996ar

DOI : https://doi.org/10.7202/014996ar

Aller au sommaire du numéro

\section{Éditeur(s)}

Association Canadienne d'Ethnologie et de Folklore

ISSN

1481-5974 (imprimé)

1708-0401 (numérique)

Découvrir la revue

Citer ce compte rendu

Banu, C. A. (2006). Compte rendu de [Regard sur... les jeunes en Europe centrale et orientale. Par Mircea Vultur, dir. (Québec : Presses de l'Université Laval et Éditions de l'IQRC, 2004. Pp. 142, ISBN 2-89224-347-5)]. Ethnologies, 28(2),

242-245. https://doi.org/10.7202/014996ar d'utilisation que vous pouvez consulter en ligne. 
Regard sur... les jeunes en Europe centrale et orientale. Par Mircea Vultur, dir. (Québec : Presses de l'Université Laval et Éditions de I'IQRC, 2004. Pp. 142, ISBN 2-89224-347-5)

L'ouvrage sur les jeunes de l'Europe centrale et orientale, dans le contexte des sociétés postcommunistes, s'inscrit parfaitement dans la thématique généreuse proposée par la collection « Regard sur la jeunesse du monde », dirigée par Madeline Gauthier. Depuis une trentaine d'années, les jeunes, qu'ils soient des pays de démocratie libérale ou des sociétés postcoloniales et post-totalitaires, leurs problèmes spécifiques et les méthodes d'analyse de cette problématique, constituent des axes privilégiés de la recherche en sciences humaines.

Cet ouvrage dirigé par Mircea Vultur, professeur d'économie et de sociologie au Centre Urbanisation, culture et société de l'Institut National de la Recherche Scientifique (INRS) de Québec et membre du comité scientifique de l'Observatoire Jeunes et Société, réunit sept études sur les jeunes de l'ancien bloc totalitaire communiste, réalisées par neuf jeunes chercheurs de cet espace : la Roumanie, la Slovaquie, la Russie. Sans prétendre être un tout unitaire, à travers ses chapitres l'ouvrage présente un inventaire des problématiques, des contextes et des méthodes d'analyse sur la jeunesse d'un espace où, jusqu'à récemment, l'idéologie communiste monolithique basée sur la théorie des «classes sociales » et des «catégories d'âge » prétendait tout rapporter à un conformisme forcé et pénible.

À l'époque de "l'univers clos » communiste, certains domaines des sciences humaines comme la sociologie et la psychologie ont été considérés comme "subversifs » et par conséquent ostracisés. La conjoncture créée par la chute du communisme est donc favorable à une démarche pionnière de "réhabilitation » de ceux-ci, afin de combler le vide laissé par un manque grave de recherches de ce type.

Les thèmes du livre (jeunes, relations familiales, travail, éducation, participation politique et civique, religion, itinéraires socioprofessionnels) sont alliés aux concepts de «transition » et de «changement». Cette problématique était déjà au coeur des préoccupations, aussi diversifiées, des jeunes chercheurs de l'Europe centrale et orientale, que ce soit en sociologie, économie, sciences politiques, éducation, ou gestion des ressources humaines. Ainsi, le coordonnateur de l'ouvrage, Mircea Vultur, est aussi l'auteur de Collectivisme et transition démocratique. Les campagnes roumaines à l'épreuve 
du marché (Presses de l'Université Laval, 2002), où cette même dynamique de la "transition » de la société postcommuniste roumaine, les déconstructions et les reconstructions identitaires traversaient déjà les pages de ce livre sur la question de l'héritage socialiste dans le comportement des paysans de la Roumanie, les grands bouleversements de toutes sortes, le rejet et l'adoption des modèles. Il a publié aussi toute une série d'articles et de chapitres dans des ouvrages collectifs portant sur les transformations postcommunistes en Europe centrale et orientale et sur l'insertion professionnelle des jeunes au Canada.

Le style de l'ouvrage est analytique et prospectif, en s'appuyant sur des données intéressantes de nature statistique ou empirique. Le matériel documentaire par spécialisations est aussi diversifié, contenant à la fois des références sur l'espace européen post-totalitaire et sur le monde de la démocratie libérale. Certaines parties sont des études de cas, tandis que d'autres sont des recherches comparatives.

Cet ouvrage captivant s'adresse à la fois aux jeunes et aux adultes initiés à la recherche en sciences humaines. Son intérêt réside dans son caractère exploratoire et empirique qui, loin d'être une faiblesse méthodologique, lui confère une originalité évidente par rapport aux recherches de même type. Les rapports sociaux, économiques, politiques, culturels, religieux, extrêmement complexes, dans quelques sociétés post-totalitaires de l'Europe centrale et orientale, avec toutes leurs ruptures, segmentations et mutations sont saisies par un regard attentif et avec une profondeur remarquable, dans des études de dimensions raisonnables et bien justifiées du point de vue de l'argumentation. L'ouvrage ne se propose pas d'ailleurs d'épuiser une si vaste thématique. Il ouvre seulement la voie à de futures recherches, dans un domaine longtemps ignoré ou étudié seulement à travers la «grille d'analyse » du « socialisme triomphant ». $\grave{A}$ travers cet ouvrage, on peut facilement constater que même si le communisme n'a pas abouti à une adhésion commune dans les pays de l'espace européen central et oriental, il a créé au moins presque les mêmes problèmes durant la période posttotalitaire en ce qui concerne les jeunes. Le ton du livre est calme, se préoccupant plutôt de l'analyse scientifique que des querelles méthodologiques ou des polémiques idéologiques post-factum, même si la «transition» et les problèmes des jeunes font actuellement l'objet de vifs débats dans les pays qui ont connu les «bienfaits»du communisme. Même si les chapitres ne suivent pas une progression linéaire de la «polygraphie de la jeunesse » (Mircea Vultur et Elena 
Fecioru), ils se complètent de façon admirable, en donnant au livre une valeur scientifique à la fois segmentaire et globale. Tout d'abord, Siyha Kovacheva aborde les formes de participation civique de la jeunesse durant la période post-totalitaire avec tous ses obstacles et conditions favorables, par tout un éventail d'exemples : projets, actions, institutions, effets, et capacité de s'associer et de résister à la pression de l'État et d'assumer la liberté. Puis Ivan Bajoumi explore les transformations du système d'enseignement et l'impact sur la formation des jeunes pour plusieurs pays de l'Europe post-totalitaire, par le démantèlement du centralisme, les jeux d'échelle (macro, micro) et l'adaptation de la mentalité, contre la tendance hégémonique de l'État.

Alexandru Gurau se penche ensuite sur la nature des rapports des jeunes de l'Europe post-totalitaire avec la religion dans le contexte d'une pluralité des offres religieuses. Son explication est la forte revalorisation de la diversité religieuse, comme lieu privilégié de contestation, d'attestation, d'insertion sociale et d'espace de refuge, autrefois interdit.

Mircea Vultur et Elena Fecioru analysent les dynamiques du désenchantement qui couvrent les divers aspects de la situation des jeunes dans la période de transition démocratique roumaine. Les auteurs réalisent un vaste diagnostic de la situation économique et sociale des jeunes, de leurs relations familiales, des problèmes d'éducation, de travail, de la mobilité, de l'émigration, de la participation politique, de l'implication civique, en essayant d'appréhender les conditions difficiles de transformation.

Julia Zubok analyse la notion de « risque»dans la société postcommuniste russe, avec des jeunes « hyper-sensibilisés » par rapport aux adultes, le risque "objectif » des rapports sociaux et le risque «subjectif», c'est-à-dire les prédispositions naturelles inconscientes. Le rapport entre les deux dimensions du risque influence le processus identitaire, les possibilités théoriques de carrière, processus qui dépend clairement des catégories des jeunes et de leur mode d'agir. Ladislav Machacek observe, dans son étude, l'ouverture des jeunes à l'autonomie individuelle en Slovaquie et exemplifie la théorie des formes générationnelles de Karl Manheim et les possibilités de mobilité sociale et spatiale par des données « longitudinales ». Enfin, Ionela Roharik réalise une analyse socioéconomique sur les caractéristiques du marché du travail des jeunes en Roumanie, sur leur profil social, les rapports 
formation-emploi, le rôle du travail dans le passage à l'âge adulte, le capitalisme, la mentalité et la construction des stratégies situationnelles.

Au fil de la lecture, on observe bien le changement de paradigme qui traverse la recherche dans le domaine de la sociologie des jeunes après la chute du communisme en Europe centrale et orientale. Ainsi, l'ouvrage propose l'illustration d'une série de concepts consacrés par des titans de la recherche sociologique comme K. Manheim. Dans une société communiste fortement caractérisée par l'atomisation du corps social, la jeunesse était circonscrite de façon arbitraire et évidemment non scientifique dans le concept de "catégorie d'âge ", concept vague, homogénéisant et formel qui prétendait résoudre d'un seul coup les problématiques complexes de la société bureaucratique et paternaliste. Au lieu de cette «simplification » superficielle, les jeunes chercheurs de l'espace post-totalitaire proposent le concept de "groupe social» qui, loin d'être une entité globale, contient des sous-groupes culturels et professionnels se distinguant par un ancrage identitaire, par une diversité de choix religieux (Alexandru Gurau) et par un degré plus ou moins élevé de risque (Julia Zubok). Il s'agit ici de petites "entités ", "d'acteurs sociaux », d'une "fragmentation de la jeunesse ", à l'échelle micro et macro, des jeunes qui "pensent, ressentent et agissent », de façon non homogène, autant dans ses structures que dans ses désirs. Les jeunes ne sont pas perçus par une analyse téléologique, mais sous l'angle dynamique des rapports des forces, des conflits et de la coopération, de la capacité d'assumer la liberté et de créer des stratégies d'adaptation individuelles et de groupe face à la pression de l'État postcommuniste (Ladislav Machacek), encore paternaliste et centralisateur (Ionela Roharik).

Pour conclure notre brève appréciation critique, cet ouvrage montre que les transformations structurelles dans l'ancien espace totalitaire supposent des stratégies diversifiées de la part des jeunes, une adaptation de ceux-ci à la rationalité capitaliste et aussi d'autres représentations de la famille, de la religion, de la politique et du travail par rapport à ce à quoi le régime communiste avait habitué la société pendant presque un demi-siècle. Cela peut certainement susciter l'intérêt du lecteur, qu'il vienne du monde «libre » ou de l'espace postcommuniste. 